\title{
Group quasi-representations and almost flat bundles
}

\author{
Marius Dadarlat*
}

\begin{abstract}
We study the existence of quasi-representations of discrete groups $G$ into unitary groups $U(n)$ that induce prescribed partial maps $K_{0}\left(C^{*}(G)\right) \rightarrow \mathbb{Z}$ on the K-theory of the group $C^{*}$-algebra of $G$. We give conditions for a discrete group $G$ under which the K-theory group of the classifying space $B G$ consists entirely of almost flat classes.
\end{abstract}

Mathematics Subject Classification (2010). 46L80, 46L65, 19K35, 19 K56.

Keywords. K-theory, discrete groups, deformations, almost flat bundles.

\section{Introduction}

The notions of almost flat bundle and group quasi-representation were introduced by Connes, Moscovici and Gromov [4] as tools for proving the Novikov conjecture for large classes of groups. The first example of a topologically nontrivial quasirepresentation is due to Voiculescu for $G=\mathbb{Z}^{2}$, [27]. In this paper we use known results on the Novikov and the Baum-Connes conjectures to derive the existence of topologically nontrivial quasi-representations of certain discrete groups $G$, as well as the existence of nontrivial almost flat bundles on the classifying space $B G$, by employing the concept of quasidiagonality.

A discrete completely positive asymptotic representation of a $\mathrm{C}^{*}$-algebra $A$ consists of a sequence $\left\{\pi_{n}: A \rightarrow \mathrm{M}_{k(n)}(\mathbb{C})\right\}_{n}$ of unital completely positive maps such that $\lim _{n \rightarrow \infty}\left\|\pi_{n}\left(a a^{\prime}\right)-\pi_{n}(a) \pi_{n}\left(a^{\prime}\right)\right\|=0$ for all $a, a^{\prime} \in A$. The sequence $\left\{\pi_{n}\right\}_{n}$ induces a unital $*$-homomorphism

$$
A \rightarrow \prod_{n} \mathrm{M}_{k(n)}(\mathbb{C}) / \sum_{n} \mathrm{M}_{k(n)}(\mathbb{C})
$$

and hence a group homomorphism $K_{0}(A) \rightarrow \prod_{n} \mathbb{Z} / \sum_{n} \mathbb{Z}$. This gives a canonical way to push forward an element $x \in K_{0}(A)$ to a sequence of integers $\left(\pi_{n \sharp}(x)\right)$, which is well-defined up to tail equivalence; two sequences are tail equivalent, $\left(y_{n}\right) \equiv\left(z_{n}\right)$, if there is $m$ such that $x_{n}=y_{n}$ for all $n \geq m$.

\footnotetext{
*The author was partially supported by NSF grant \#DMS-1101 305.
} 
In the first part of the paper we study the existence of discrete asymptotic representations of group $\mathrm{C}^{*}$-algebras that interpolate on $\mathrm{K}$-theory a given group homomorphism $h: K_{0}\left(C^{*}(G)\right) \rightarrow \mathbb{Z}$. We rely heavily on results of Kasparov, Higson, $\mathrm{Yu}$, Skandalis and Tu [15], [12], [29], [24], [16], [26]. For illustration, we have the following:

Theorem 1.1. Let $G$ be a countable, discrete, torsion-free group with the Haagerup property. Suppose that $C^{*}(G)$ is residually finite dimensional. Then, for any group homomorphism $h: K_{0}\left(C^{*}(G)\right) \rightarrow \mathbb{Z}$, there is a discrete completely positive asymptotic representation $\left\{\pi_{n}: C^{*}(G) \rightarrow \mathrm{M}_{k(n)}(\mathbb{C})\right\}_{n}$ such that $\pi_{n \sharp}(x) \equiv h(x)$ for all $x \in K_{0}(I(G))$.

Here $I(G)$ is the kernel of the trivial representation $\iota: C^{*}(G) \rightarrow \mathbb{C}$. By contrast, any finite dimensional unitary representation of $G$ induces the zero map on $K_{0}(I(G))$. The groups with the Haagerup property are characterized by the requirement that there exists a sequence of normalized continuous positive-definite functions which vanish at infinity on $\mathrm{G}$ and converge to 1 uniformly on finite subsets of $\mathrm{G}$. The conclusion of Theorem 1.1 also holds if $G$ is an increasing union of residually finite amenable groups, see Theorem 3.4. The class of groups considered in Theorem 1.1 contains all countable, torsion-free, amenable, residually finite groups (also the maximally periodic groups) and the surface groups [17]. Moreover, this class is closed under free products (see [10], [3]). If we impose a weaker condition, namely that $C^{*}(G)$ is quasidiagonal, then in general we need two asymptotic representations in order to interpolate $h$, see Theorem 3.3. Theorem 1.1 remains true if we replace the assumption that $G$ has the Haagerup property by the requirements that $G$ is uniformly embeddable in a Hilbert space and that the assembly map $\mu: \mathrm{RK}_{0}(\mathrm{~B} G) \rightarrow K_{0}\left(C^{*}(G)\right)$ is surjective. Let us recall that Hilbert space uniform embeddability of $G$ implies that $\mu$ is split injective, as proven by $\mathrm{Yu}$ [29] if the classifying space $\mathrm{B} G$ is finite and by Skandalis, $\mathrm{Yu}$ and $\mathrm{Tu}$ [24] in the general case. We will also use a strengthening of this result by $\mathrm{Tu}[26]$ who showed that $G$ has a gamma element. In conjunction with a theorem of Kasparov [15] this guarantees the surjectivity of the dual assembly map $\nu: K^{0}\left(C^{*}(G)\right) \rightarrow \mathrm{RK}^{0}(\mathrm{~B} G)$ for countable, discrete, torsion-free groups which are uniformly embeddable in a Hilbert space.

The notion of almost flat K-theory class was introduced in [4] as a tool for proving the Novikov conjecture. In the second part of the paper we pursue a reverse direction. Namely, we use known results on the Baum-Connes and the Novikov conjectures to derive the existence of almost flat K-theory classes by employing the concept of quasidiagonality.

Theorem 1.2. Let $G$ be a countable, discrete, torsion-free group which is uniformly embeddable in a Hilbert space. Suppose that the classifying space $\mathrm{B} G$ is a finite simplicial complex and that the full group $C^{*}$-algebra $C^{*}(G)$ is quasidiagonal. Then all the elements of $K^{0}(\mathrm{~B} G)$ are almost flat. 
The class of groups considered in Theorem 1.2 is closed under free products, by [1] and [2]. If $G$ can be written as a union of amenable residually finite groups (as is the case if $G$ is a linear amenable group), then $C^{*}(G)$ is quasidiagonal. It is an outstanding open question if all discrete amenable groups have quasidiagonal $\mathrm{C}^{*}$-algebras [28].

Voiculescu has asked in [28] if there are invariants of a topological nature which can be used to describe the obstruction that a $\mathrm{C}^{*}$-algebra be quasidiagonal. One can view Theorem 1.2 as further evidence towards a topological nature of quasidiagonality, since it shows that the existence of non-almost flat classes in $K^{0}(\mathrm{~B} G)$ represents an obstruction for the quasidiagonality of $C^{*}(G)$.

The fundamental connection between deformations of $\mathrm{C}^{*}$-algebras and $\mathrm{K}$-theory was discovered by Connes and Higson [5]. They introduced the concept of asymptotic homomorphism of $\mathrm{C}^{*}$-algebras which formalizes the intuitive idea of deformations of $\mathrm{C}^{*}$-algebras. An asymptotic homomorphism is a family of maps $\varphi_{t}: A \rightarrow B$, $t \in[0, \infty)$, such that for each $a \in A$ the map $t \rightarrow \varphi_{t}(a)$ is continuous and bounded and the family $\left(\varphi_{t}\right)_{t \in[0, \infty)}$ satisfies asymptotically the axioms of $*$-homomorphisms. There is a natural notion of homotopy for asymptotic homomorphisms. E-theory is defined as homotopy classes of asymptotic homomorphisms from the suspension of $A$ to the stable suspension of $B, E(A, B)=\llbracket C_{0}(\mathbb{R}) \otimes A, C_{0}(\mathbb{R}) \otimes B \otimes \mathcal{K} \rrbracket$. The introduction of the suspension and of the compact operators $\mathcal{K}$ yields an abelian group structure on $E(A, B)$. Connes and Higson showed that E-theory defines the universal half-exact $\mathrm{C}^{*}$-stable homotopy functor on separable $\mathrm{C}^{*}$-algebras. In particular the KK-theory of Kasparov factors through E-theory. A similar construction based on completely positive asymptotic homomorphisms gives a realization of KK-theory itself as shown by Larsen and Thomsen [13].

While E-theory gives in general maps of suspensions of $\mathrm{C}^{*}$-algebras it is often desirable to have interesting deformations of unsuspended $\mathrm{C}^{*}$-algebras. In joint work with Loring [8], [6], we proved a suspension theorem for commutative $\mathrm{C}^{*}$ algebras $A=C_{0}\left(X \backslash x_{0}\right)$, where $X$ is a compact connected space and $x_{0} \in X$ is a base point. Specifically, we showed that the reduced K-homology group $\widetilde{K}_{0}(X)=$ $K_{0}\left(X, x_{0}\right)$ is isomorphic to the homotopy classes of asymptotic homomorphisms $\llbracket C_{0}\left(X \backslash x_{0}\right), \mathcal{K} \rrbracket$. One can replace the compact operators $\mathcal{K}$ by $\bigcup_{n=1}^{\infty} \mathrm{M}_{n}(\mathbb{C})$ and conclude that the reduced K-homology of $X$ classifies the deformations of $C_{0}(X)$ into matrices. The case of $X=\mathbb{T}^{2}$ played an important role in the history of the subject. Indeed, Voiculescu [27] exhibited pairs of almost commuting unitaries $u, v \in U(n)$ whose properties reflect the non-triviality of $H^{2}\left(\mathbb{T}^{2}, \mathbb{Z}\right)$. One can view such a pair as associated to a quasi-representation of $C^{*}\left(\mathbb{Z}^{2}\right) \cong C\left(\mathbb{T}^{2}\right)$. If the commutator $\|u v-v u\|$ is sufficiently small, then there is an induced pushforward of the Bott class that represents the obstruction for perturbing $u, v$ to a pair of commuting unitaries, [27], [9]. It is therefore quite natural to investigate deformations of $\mathrm{C}^{*}$ algebras associated to non-commutative groups. In view of Theorem 1.1 we propose the following: 
Conjecture. If $G$ is a discrete, countable, torsion-free, amenable group, then the natural map $\llbracket I(G), \mathcal{K} \rrbracket \rightarrow \mathrm{KK}(I(G), \mathcal{K}) \cong K^{0}(I(G))$ is an isomorphism of groups.

This is verified if $G$ is commutative. Indeed, $I(G) \cong C_{0}\left(\hat{G} \backslash x_{0}\right)$ and $\widehat{G}$ is connected since $G$ is torsion-free, so that we can apply the suspension result of [6].

Manuilov, Mishchenko and their co-authors have studied various aspects and applications of quasi-representations and asymptotic representations of discrete groups. The paper [18] is a very interesting survey of their contributions. The notion of quasirepresentation of a group is used in the literature in several non-equivalent contexts, to mean several different things, see [22].

\section{Quasi-representations and K-theory}

Definition 2.1. Let $A$ and $B$ be unital $\mathrm{C}^{*}$-algebras. Let $F \subset A$ be a finite set and let $\varepsilon>0$. A unital completely positive map $\varphi: A \rightarrow B$ is called an $(F, \varepsilon)$ homomorphism if $\left\|\varphi\left(a a^{\prime}\right)-\varphi(a) \varphi\left(a^{\prime}\right)\right\|<\varepsilon$ for all $a, a^{\prime} \in F$. If $B$ is the $\mathrm{C}^{*_{-}}$ algebra of bounded linear operators on a Hilbert space, then we say that $\varphi$ is an $(F, \varepsilon)$-representation of $A$. We will use the term quasi-representation to refer to an $(F, \varepsilon)$-representation where $F$ and $\varepsilon$ are not necessarily specified.

An important method for turning K-theoretical invariants of $A$ into numerical invariants is to use quasi-representations to pushforward projections in matrices over $A$ to scalar projections. Consider a finite set of projections $\mathcal{P} \subset \mathrm{M}_{m}(A)$. We say that $(\mathcal{P}, F, \varepsilon)$ is a $K_{0}$-triple if for any $(F, \varepsilon)$-homomorphism $\varphi: A \rightarrow B$ and $p \in \mathcal{P}$, the element $b=\left(\operatorname{id}_{m} \otimes \varphi\right)(p)$ satisfies $\left\|b^{2}-b\right\|<1 / 4$ and hence the spectrum $\operatorname{sp}(b)$ of $b$ is contained in $[0,1 / 2) \cup(1 / 2,1]$. We denote by $q$ the projection $\chi(b)$, where $\chi$ is the characteristic function of the interval $(1 / 2,1]$. It is not hard to show that for any finite set of projections $\mathcal{P}$ there exist a finite set $F \subset A$ and $\varepsilon>0$ such that $(\mathcal{P}, F, \varepsilon)$ is a $K_{0}$-triple. If $(\mathcal{P}, F, \varepsilon)$ is a $K_{0}$-triple, then any $(F, \varepsilon)$-homomorphism $\varphi: A \rightarrow B$ induces a map $\varphi_{\sharp}: \mathcal{P} \rightarrow K_{0}(B)$ defined by $\varphi_{\sharp}(p)=[q]$. Let $\operatorname{Proj}(A)$ denote the set of all projections in matrices over $A$. It is convenient to extend $\varphi_{\sharp}$ to $\operatorname{Proj}(A)$ by setting $\varphi_{\sharp}(p)=0$ if $b=\left(\operatorname{id}_{m} \otimes \varphi\right)(p)$ does not satisfy $\left\|b^{2}-b\right\|<1 / 4$. If $\varphi$ were a $*$-homomorphism, then $\varphi$ would induce a map $\varphi_{*}: K_{0}(A) \rightarrow K_{0}(B)$. Intuitively, one may think of $\varphi_{\sharp}$ as a substitute for $\varphi_{*}$.

Two sequences $\left(a_{n}\right)$ and $\left(b_{n}\right)$ are called tail-equivalent if there is $n_{0}$ such that $a_{n}=b_{n}$ for $n \geq n_{0}$. Tail-equivalence is denoted by $\left(a_{n}\right) \equiv\left(b_{n}\right)$ or even $a_{n} \equiv b_{n}$, abusing the notation.

We will also work with discrete completely positive asymptotic morphisms $\left(\varphi_{n}\right)_{n}$. They consists of a sequence of contractive completely positive maps $\varphi_{n}: A \rightarrow B_{n}$ with $\lim _{n \rightarrow \infty}\left\|\varphi_{n}\left(a a^{\prime}\right)-\varphi_{n}(a) \varphi_{n}\left(a^{\prime}\right)\right\|=0$ for all $a, a^{\prime} \in A$. If in addition each $B_{n}$ is a matricial algebra $B_{n}=\mathrm{M}_{k(n)}(\mathbb{C})$, then we call $\left(\varphi_{n}\right)_{n}$ a discrete asymptotic representation of $A$. A discrete completely positive asymptotic morphism $\left(\varphi_{n}\right)_{n}$ 
induces a sequence of maps $\varphi_{n \sharp}: \operatorname{Proj}(A) \rightarrow K_{0}\left(B_{n}\right)$. Note that if $p, q \in \operatorname{Proj}(A)$ have the same class in $K_{0}(A)$, then $\varphi_{n \sharp}(p) \equiv \varphi_{n \sharp}(q)$.

For any $x \in K_{0}(A)$, we fix projections $p, q \in \operatorname{Proj}(A)$ such that $x=[p]-[q]$ and set $\varphi_{n \sharp}(x)=\varphi_{n \sharp}(p)-\varphi_{n \sharp}(q) \in K_{0}\left(B_{n}\right)$. The sequence $\left(\varphi_{n \sharp}(x)\right)$ depends on the particular projections that we use to represent $x$ but only up to tail-equivalence. While in general the maps $\varphi_{n \sharp}: K_{0}(A) \rightarrow K_{0}\left(B_{n}\right)$ are not group homomorphisms, the sequence $\left(\varphi_{n \sharp}(x)\right)$ does satisfy $\left(\varphi_{n \sharp}(x+y)\right) \equiv\left(\varphi_{n \sharp}(x)+\varphi_{n \sharp}(y)\right)$ for all $x, y \in$ $K_{0}(A)$.

A subset $B \subset L(H)$ is called quasidiagonal if there is an increasing sequence $\left(p_{n}\right)$ of finite rank projections in $L(H)$ which converges strongly to $1_{H}$ and such that $\lim _{n \rightarrow \infty}\left\|\left[b, p_{n}\right]\right\|=0$ for all $b \in B . B$ is block-diagonal if there is a sequence $\left(p_{n}\right)$ as above such that $\left[b, p_{n}\right]=0$ for all $b \in B$ and $n \geq 1$. Let $A$ be a separable $\mathrm{C}^{*}$-algebra. Let us recall that the elements of $\operatorname{KK}(A, \mathbb{C})$ can be represented by Cuntz pairs, i.e., by pair of $*$-representations $\varphi, \psi: A \rightarrow L(H)$ such that $\varphi(a)-\psi(a) \in$ $K(H)$ for all $a \in A$.

Definition 2.2. Let $A$ be a separable $\mathrm{C}^{*}$-algebra. An element $\alpha \in \mathrm{KK}(A, \mathbb{C})$ is called quasidiagonal if it can be represented by a Cuntz pair $(\varphi, \psi): A \rightarrow L(H)$ with the property that the set $\psi(A) \subset L(H)$ is quasidiagonal. In this case let us note that the set $\varphi(A) \subset L(H)$ must be also quasidiagonal. Similarly, we say that $\alpha$ is residually finite dimensional if it can be represented by a Cuntz pair with the property that the set $\psi(A)$ is block-diagonal. We denote by $\operatorname{KK}_{\mathrm{qd}}(A, \mathbb{C})$ the subset of $\operatorname{KK}(A, \mathbb{C})$ consisting of quasidiagonal classes and by $\operatorname{KK}_{\mathrm{rfd}}(A, \mathbb{C})$ the subset of $\mathrm{KK}(A, \mathbb{C})$ consisting of residually finite dimensional classes. It is clear that $\mathrm{KK}_{\mathrm{rfd}}(A, \mathbb{C}) \subset \mathrm{KK}_{\mathrm{qd}}(A, \mathbb{C})$, that $\mathrm{KK}_{\mathrm{qd}}(A, \mathbb{C})$ is a subgroup of $\mathrm{KK}(A, \mathbb{C})$ and that $\mathrm{KK}_{\mathrm{rfd}}(A, \mathbb{C})$ is a subsemigroup.

We say that $A$ is $\mathrm{K}$-quasidiagonal if $\mathrm{KK}_{\mathrm{qd}}(A, \mathbb{C})=\mathrm{KK}(A, \mathbb{C})$ and that $A$ is $\mathrm{K}$-residually finite dimensional if $\mathrm{KK}_{\mathrm{rfd}}(A, \mathbb{C})=\mathrm{KK}(A, \mathbb{C})$.

Remark 2.3. Let $A$ be a separable $\mathrm{C}^{*}$-algebra. It was pointed out by Skandalis [23] that for any given faithful $*$-representation $\pi: A \rightarrow L(H)$ such that $\pi(A) \cap K(H)=$ $\{0\}$, one can represent all the elements of $\operatorname{KK}(A, \mathbb{C})$ by Cuntz pairs where the second map is fixed and equal to $\pi$. It follows that a separable quasidiagonal $\mathrm{C}^{*}$-algebra is $\mathrm{K}$-quasidiagonal and a separable residually finite dimensional $\mathrm{C}^{*}$-algebra is $\mathrm{K}$ residually finite dimensional. More generally, if $A$ is homotopically dominated by $B$ and $B$ is $\mathrm{K}$-quasidiagonal or $\mathrm{K}$-residually finite dimensional then so is $A$. Let us note that the Cuntz algebra $\mathrm{O}_{2}$ is $\mathrm{K}$-residually finite dimensional while it is not quasidiagonal.

The following lemma and proposition are borrowed from [7]. For the sake of completeness, we review briefly some of the arguments from their proofs. Let $B$ be a unital $\mathrm{C}^{*}$-algebra and let $E$ be a right Hilbert $B$-module. If $e, f \in L_{B}(E)$ are projections such that $e-f \in K_{B}(E)$, we denote by $[e, f]$ the corresponding element of $\mathrm{KK}(\mathbb{C}, B) \cong K_{0}(B)$. 
Lemma 2.4. Let $B$ be a unital $C^{*}$-algebra and let $E$ be a right Hilbert $B$-module. Let $e, f \in L_{B}(E)$ and $h \in K_{B}(E)$ be projections such that $e-f \in K_{B}(E)$ and $\|e h-h e\| \leq 1 / 9,\|f h-h f\| \leq 1 / 9,\|(1-h)(e-f)(1-h)\| \leq 1 / 9$. Then

$$
\begin{gathered}
\operatorname{sp}(h e h) \cup \operatorname{sp}(h f h) \subset[0,1 / 2) \cup(1 / 2,1], \\
{[e, f]=[\chi(h e h), \chi(h f h)] \in \mathrm{KK}(\mathbb{C}, B) \cong K_{0}(B) .}
\end{gathered}
$$

Proof. One shows that if $e^{\prime}, f^{\prime} \in L_{B}(E)$ are projections such that $e^{\prime}-f^{\prime} \in K_{B}(E)$ and $\left\|e-e^{\prime}\right\|<1 / 2,\left\|f-f^{\prime}\right\|<1 / 2$, then $[e, f]=\left[e^{\prime}, f^{\prime}\right]$. This is proved using the homotopy $\left(\chi\left(e_{t}\right), \chi\left(f_{t}\right)\right)$ where $e_{t}=(1-t) e+t e^{\prime}, f_{t}=(1-t) f+t f^{\prime}, 0 \leq t \leq 1$. Then one applies this observation to conclude that

$[e, f]=\left[\chi(x)+\chi\left(x^{\prime}\right), \chi(y)+\chi\left(y^{\prime}\right)\right]=\left[\chi(x)+\chi\left(x^{\prime}\right), \chi(y)+\chi\left(x^{\prime}\right)\right]=[\chi(x), \chi(y)]$,

where $x=h e h, x^{\prime}=(1-h) e(1-h), y=h f h, y^{\prime}=(1-h) f(1-h)$.

Let $A, B$ be separable $\mathrm{C}^{*}$-algebras. An element $\alpha \in \mathrm{KK}(A, \mathbb{C})$ induces a group homomorphism $\alpha_{*}: K_{0}(A \otimes B) \rightarrow K_{0}(B)$ via the cup product

$$
\mathrm{KK}(\mathbb{C}, A \otimes B) \times \mathrm{KK}(A, \mathbb{C}) \rightarrow \mathrm{KK}(\mathbb{C}, B), \quad(x, \alpha) \mapsto x \circ\left(\alpha \otimes 1_{B}\right) .
$$

Here we work with the maximal tensor product.

Proposition 2.5. Let $A$ be a separable unital $C^{*}$-algebra and $\alpha \in \mathrm{KK}_{\mathrm{qd}}(A, \mathbb{C})$. There exist two discrete asymptotic representations $\left(\varphi_{n}\right)_{n}$ and $\left(\psi_{n}\right)_{n}$ consisting of unital completely positive maps $\varphi_{n}: A \rightarrow \mathrm{M}_{k(n)}(\mathbb{C})$ and $\psi_{n}: A \rightarrow \mathrm{M}_{r(n)}(\mathbb{C})$ such that for any separable unital $C^{*}$-algebra $B$, the map $\alpha_{*}: K_{0}(A \otimes B) \rightarrow K_{0}(B)$ has the property that

$$
\alpha_{*}(x) \equiv\left(\varphi_{n} \otimes \operatorname{id}_{B}\right)_{\sharp}(x)-\left(\psi_{n} \otimes \operatorname{id}_{B}\right)_{\sharp}(x)
$$

for all $x \in K_{0}(A \otimes B)$. If $\alpha \in \mathrm{KK}_{\mathrm{rfd}}(A, \mathbb{C})$, then all $\psi_{n}$ can be chosen to be *-representations.

Proof. Represent $\alpha$ by a Cuntz pair $\varphi, \psi: A \rightarrow L(H)$ with $\varphi(a)-\psi(a) \in K(H)$, for all $a \in A$, and such that the set $\psi(A)$ is quasidiagonal. Therefore there is an increasing approximate unit $\left(p_{n}\right)_{n}$ of $K(H)$ consisting of projections such that $\left(p_{n}\right)_{n}$ commutes asymptotically with both $\varphi(A)$ and $\psi(A)$. Let us define contractive completely positive maps $\varphi_{n}, \psi_{n}: A \rightarrow L\left(p_{n} H\right)$ by $\varphi_{n}(a):=p_{n} \varphi(a) p_{n}$ and $\psi_{n}(a):=p_{n} \psi(a) p_{n}$. Without any loss of generality we may assume that $x$ is the class of a projection $e \in A \otimes B$. It follows from the definition of the Kasparov product that

$$
\alpha_{*}(x)=\left[\left(\varphi \otimes \operatorname{id}_{B}\right)(e),\left(\psi \otimes \operatorname{id}_{B}\right)(e)\right] \in \mathrm{KK}(\mathbb{C}, B) .
$$


On the other hand, the sequence of projections $p_{n} \otimes 1_{B} \in K(H) \otimes B$ commutes asymptotically with both projections $\left(\varphi \otimes \mathrm{id}_{B}\right)(e)$ and $\left(\psi \otimes \mathrm{id}_{B}\right)(e)$ and moreover

$$
\lim _{n \rightarrow \infty}\left\|p_{n} \otimes 1_{B}\left(\left(\varphi \otimes \operatorname{id}_{B}\right)(e)-\left(\psi \otimes \operatorname{id}_{B}\right)(e)\right) p_{n} \otimes 1_{B}\right\|=0,
$$

since the sequence $\left(p_{n} \otimes 1_{B}\right)_{n}$ forms an approximative unit of $K(H) \otimes B$. Now it follows from Lemma 2.4 that

$$
\left[\left(\varphi \otimes \operatorname{id}_{B}\right)(e),\left(\psi \otimes \operatorname{id}_{B}\right)(e)\right]=\left(\varphi_{n} \otimes \operatorname{id}_{B}\right)_{\sharp}(e)-\left(\psi_{n} \otimes \operatorname{id}_{B}\right)_{\sharp}(e)
$$

for all sufficiently large $n$. It is standard to perturb $\varphi_{n}$ and $\psi_{n}$ to completely positive maps such that $\varphi_{n}(1)$ and $\psi_{n}(1)$ are projections. Finally, let us note that $\psi_{n}$ is a $*$-homomorphism if $p_{n}$ commutes with $\psi$.

\section{Asymptotic representations of group $\mathrm{C}^{*}$-algebras}

We use the following notation for the Kasparov product:

$$
\mathrm{KK}(A, B) \times \mathrm{KK}(B, C) \rightarrow \mathrm{KK}(A, C), \quad(y, x) \mapsto y \circ x .
$$

In the case of the pairing $K_{i}(B) \times K^{i}(B) \rightarrow \mathbb{Z}$ we will also write $\langle y, x\rangle$ for $y \circ x$. We are mostly interested in the map

$$
K^{i}\left(C^{*}(G)\right) \rightarrow \operatorname{Hom}\left(K_{i}\left(C^{*}(G)\right), \mathbb{Z}\right),
$$

induced by the pairing above for $B=C^{*}(G)$. If $G$ has the Haagerup property, then it was shown in [25] that $C^{*}(G)$ is KK-equivalent with a commutative $\mathrm{C}^{*}$-algebra and hence the map (1) is surjective. Assuming that $G$ is a countable, discrete, torsion-free group that is uniformly embeddable in a Hilbert space, we are going to verify that the map (1) is split surjective whenever the assembly map $\mu: \mathrm{RK}_{i}(\mathrm{~B} G) \rightarrow K_{i}\left(C^{*}(G)\right)$ is surjective.

Following Kasparov [15], for a locally compact, $\sigma$-compact, Hausdorff space $X$ and $C_{0}(X)$-algebras $A$ and $B$ we consider the representable K-homology groups $\mathrm{RK}_{i}(X)$, the representable $\mathrm{K}$-theory groups $\mathrm{RK}^{i}(X)$ and the bivariant theory $\mathcal{R K K}_{i}(X ; A, B)$. If $Y$ is compact, then $\operatorname{RK}_{i}(Y)=\mathrm{KK}_{i}(C(Y), \mathbb{C})$ and $\operatorname{RK}^{i}(Y)=$ $\mathrm{KK}_{i}(\mathbb{C}, C(Y))$. Suppose now that $X$ is locally compact, $\sigma$-compact and Hausdorff. Then

$$
\mathrm{RK}_{i}(X) \cong \underset{Y \subset X}{\lim } \mathrm{RK}_{i}(Y)=\underset{Y \subset X}{\underset{Y \subset}{\lim }} \mathrm{KK}_{i}(C(Y), \mathbb{C}),
$$

where $Y$ runs over the compact subsets of $X$. Kasparov [15], Prop. 2.20, has shown that

$$
\mathrm{RK}^{i}(X) \cong \mathcal{R K K}\left(X ; C_{0}(X), C_{0}(X)\right) .
$$


Moreover, if $Y \subset X$ is a compact set, then the restriction map $\operatorname{RK}^{i}(X) \rightarrow \mathrm{RK}^{i}(Y)$ corresponds to the map

$$
\mathcal{R K K}_{i}\left(X ; C_{0}(X), C_{0}(X)\right) \rightarrow \mathcal{R K K}(Y ; C(Y), C(Y)) \cong \mathrm{KK}_{i}(\mathbb{C}, C(Y)) .
$$

It is useful to introduce the group

$$
L K^{i}(X)=\lim _{Y \subset X} \operatorname{RK}^{i}(Y)
$$

where $Y$ runs over the compact subsets of $X$. If $X$ is written as the union of an increasing sequence $\left(Y_{n}\right)_{n}$ of compact subspaces, then, as explained in the proof of Lemma 3.4 from [16], there is a Milnor $\lim ^{1}$ exact sequence:

$$
0 \rightarrow \lim ^{1} \mathrm{RK}^{i+1}\left(Y_{n}\right) \rightarrow \mathrm{RK}^{i}(X) \rightarrow \underset{\leftarrow}{\lim } \mathrm{RK}^{i}\left(Y_{n}\right) \rightarrow 0 .
$$

The morphism $\mathrm{RK}^{i}(X) \rightarrow \operatorname{Hom}\left(\mathrm{RK}_{i}(X), \mathbb{Z}\right)$ induced by the pairing $\mathrm{RK}_{i}(X) \times$ $\mathrm{RK}^{i}(X) \rightarrow \mathbb{Z}$ factors through the morphism

$$
\begin{aligned}
\lim _{\leftarrow} \operatorname{RK}^{i}\left(Y_{n}\right)=L K^{i}(X) \rightarrow \operatorname{Hom}\left(\operatorname{RK}_{i}(X), \mathbb{Z}\right) & =\operatorname{Hom}\left(\stackrel{\left.\lim \operatorname{RK}_{i}\left(Y_{n}\right), \mathbb{Z}\right)}{\longrightarrow}\right. \\
& \cong \underset{\leftarrow}{\lim } \operatorname{Hom}\left(\operatorname{RK}_{i}\left(Y_{n}\right), \mathbb{Z}\right)
\end{aligned}
$$

given by the projective limit of the morphisms $\operatorname{RK}^{i}\left(Y_{n}\right) \rightarrow \operatorname{Hom}\left(\operatorname{RK}_{i}\left(Y_{n}\right), \mathbb{Z}\right)$.

If $X$ is a locally finite separable $C W$-complex, then there is a Universal Coefficient Theorem [16], Lemma 3.4:

$$
0 \rightarrow \operatorname{Ext}\left(\mathrm{RK}_{i+1}(X), \mathbb{Z}\right) \rightarrow \mathrm{RK}^{i}(X) \rightarrow \operatorname{Hom}\left(\mathrm{RK}_{i}(X), \mathbb{Z}\right) \rightarrow 0 .
$$

In particular, it follows that the map $L K^{i}(X) \rightarrow \operatorname{Hom}\left(\mathrm{RK}_{i}(X), \mathbb{Z}\right)$ is surjective.

Let us recall the construction of the assembly map $\mu: \mathrm{RK}_{i}(\mathrm{~B} G) \rightarrow K_{i}\left(C^{*}(G)\right)$ and of the dual map $v: K^{i}\left(C^{*}(G)\right) \rightarrow \mathrm{RK}^{i}(\mathrm{~B} G)$ as given in [15]. Kasparov considers a natural element

$$
\beta_{G} \in \mathcal{R K K}\left(\mathrm{B} G ; C_{0}(\mathrm{~B} G), C_{0}(\mathrm{~B} G) \otimes C^{*}(G)\right)
$$

(which we denote here by $\ell$ as it corresponds to Mischenko's "line bundle" on $\mathrm{B} G$ ). If $G$ is a discrete countable group then it is known [15], §6, that $\mathrm{E} G$ and $\mathrm{B} G$ can be realized as locally finite separable $\mathrm{CW}$-complexes. Write $\mathrm{B} G$ as the union of an increasing sequence $\left(Y_{n}\right)_{n}$ of finite $\mathrm{CW}$-subcomplexes. Let $\ell_{n}$ be the image of $\ell$ in

$$
\mathcal{R K K}\left(Y_{n} ; C\left(Y_{n}\right), C\left(Y_{n}\right) \otimes C^{*}(G)\right) \cong \mathrm{KK}\left(\mathbb{C}, C\left(Y_{n}\right) \otimes C^{*}(G)\right)
$$

under the restriction map induced by the inclusion $Y_{n} \subset \mathrm{B} G$.

The map $\mu_{n}: \operatorname{RK}_{i}\left(Y_{n}\right) \rightarrow K_{i}\left(C^{*}(G)\right)$ is defined as the cap product by $\ell_{n}$ :

$$
\begin{gathered}
\mathrm{KK}\left(\mathbb{C}, C\left(Y_{n}\right) \otimes C^{*}(G)\right) \times \mathrm{KK}_{i}\left(C\left(Y_{n}\right), \mathbb{C}\right) \rightarrow \mathrm{KK}_{i}\left(\mathbb{C}, C^{*}(G)\right), \\
\left(\ell_{n}, z\right) \mapsto \mu_{n}(z)=\ell_{n} \circ(z \otimes 1) .
\end{gathered}
$$


The assembly map $\mu: \mathrm{RK}_{i}(\mathrm{~B} G) \rightarrow K_{i}\left(C^{*}(G)\right)$ is the inductive limit homomorphism $\mu:=\lim _{\longrightarrow} \mu_{n}$. The homomorphism $v: K^{i}\left(C^{*}(G)\right) \rightarrow \mathrm{RK}^{i}(\mathrm{~B} G)$ is defined as the cap product by $\ell$ :

$$
\begin{gathered}
\mathcal{R K K}\left(\mathrm{B} G ; C_{0}(\mathrm{~B} G), C_{0}(\mathrm{~B} G) \otimes C^{*}(G)\right) \times \mathrm{KK}_{i}\left(C^{*}(G), \mathbb{C}\right) \\
\longrightarrow \mathcal{R K K}\left(\mathrm{B} G ; C_{0}(\mathrm{~B} G), C_{0}(\mathrm{~B} G)\right), \\
(\ell, x) \mapsto v(x)=\ell \circ(1 \otimes x) .
\end{gathered}
$$

Let $v_{n}: K^{i}\left(C^{*}(G)\right) \rightarrow \mathrm{RK}^{i}\left(Y_{n}\right)$ be obtained by composing $v$ with the restriction map $\mathrm{RK}^{i}(\mathrm{~B} G) \rightarrow \mathrm{RK}^{i}\left(Y_{n}\right)$. Noting that $\nu_{n}$ is also given by the cap product by $\ell_{n}$, Kasparov has shown that

$$
v_{n}(x) \circ z=\mu_{n}(z) \circ x
$$

for all $x \in K^{i}\left(C^{*}(G)\right)$ and $z \in \mathrm{RK}_{i}\left(Y_{n}\right)$, [15], Lemma 6.2. The assembly map induces a homomorphism $\mu^{*}: \operatorname{Hom}\left(K_{i}\left(C^{*}(G)\right), \mathbb{Z}\right) \rightarrow \operatorname{Hom}\left(\mathrm{RK}_{i}(\mathrm{~B} G), \mathbb{Z}\right)$. Since

$$
\operatorname{Hom}\left(\mathrm{RK}_{i}(\mathrm{~B} G), \mathbb{Z}\right) \cong \operatorname{Hom}\left(\lim _{\longrightarrow} \mathrm{RK}_{i}\left(Y_{n}\right), \mathbb{Z}\right) \cong \lim \operatorname{Hom}\left(\mathrm{RK}_{i}\left(Y_{n}\right), \mathbb{Z}\right)
$$

and since the equalities $v_{n}(x) \circ z=x \circ \mu_{n}(z)$ are compatible with the maps induced by the inclusions $Y_{n} \subset Y_{n+1}$, we obtain that the following diagram is commutative:

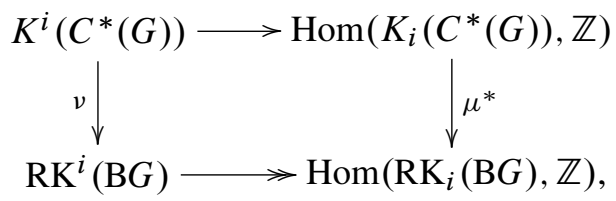

where the horizontal arrows correspond to natural pairings of K-theory with $\mathrm{K}$ homology. The map $\mathrm{RK}^{i}(\mathrm{~B} G) \rightarrow \operatorname{Hom}\left(\mathrm{RK}_{i}(\mathrm{~B} G), \mathbb{Z}\right)$ is surjective by (2).

In view of the previous discussion, by combining results of Kasparov [15] and Tu [26], one derives the following.

Theorem 3.1. Let $G$ be a countable, discrete, torsion-free group. Suppose that $G$ is uniformly embeddable in a Hilbert space. Then for any group homomorphism $h: K_{i}\left(C^{*}(G)\right) \rightarrow \mathbb{Z}$ there is $x \in K^{i}\left(C^{*}(G)\right)$ such that $h(\mu(z))=\langle\mu(z), x\rangle$ for all $z \in \mathrm{RK}_{i}(\mathrm{~B} G)$.

Proof. For a discrete group $G$ which admits a uniform embedding into a Hilbert space it was shown in [26], Thm. 3.3, that $G$ has a $\gamma$-element. Since $G$ is torsion-free, we can take $\underline{\mathrm{B}} G=\mathrm{B} G$. If $G$ has a $\gamma$-element, it follows by Theorem 6.5 and Lemma. 6.2 of [15] that the dual map $v: \mathrm{KK}_{i}\left(C^{*}(G), \mathbb{C}\right) \rightarrow \mathrm{RK}^{i}(\mathrm{~B} G)$ is split surjective. Therefore, in the diagram above, the composite map $K^{i}\left(C^{*}(G)\right) \rightarrow \operatorname{Hom}\left(\mathrm{RK}_{i}(\mathrm{~B} G), \mathbb{Z}\right)$, $x \mapsto\langle v(x), \cdot\rangle$ is surjective. This shows that if $h: K_{i}\left(C^{*}(G)\right) \rightarrow \mathbb{Z}$ is a group homomorphism, then $\mu^{*}(h)=h \circ \mu=\langle v(x), \cdot\rangle$ for some $x \in K^{i}\left(C^{*}(G)\right)$. Since the diagram above is commutative, we obtain that $h \circ \mu=\langle v(x), \cdot\rangle=\langle\mu(\cdot), x\rangle$. 
The following proposition is more or less known; for example, it is implicitly contained in [11]. Let $\iota$ be the trivial representation of $G, \iota(s)=1$ for all $s \in G$.

Proposition 3.2. Let $\mu: \mathrm{RK}_{0}(\mathrm{~B} G) \rightarrow K_{0}\left(C^{*}(G)\right)$ be the assembly map. Then $\pi_{*} \circ \mu=m \cdot \iota_{*} \circ \mu$ for any unital finite dimensional representation $\pi: C^{*}(G) \rightarrow$ $\mathrm{M}_{m}(\mathbb{C})$.

Proof. Write BG as the union of an increasing sequence $\left(Y_{n}\right)_{n}$ of finite $\mathrm{CW}$-subcomplexes. Let $z \in \operatorname{RK}_{0}\left(Y_{n}\right)$ for some $n \geq 1$ and let $x=[\pi] \in K^{0}\left(C^{*}(G)\right)$. The equality $v_{n}(x) \circ z=\mu_{n}(z) \circ x$ becomes $\left\langle v_{n}(x), z\right\rangle=\pi_{*}\left(\mu_{n}(z)\right)$. The Chern character makes the following commutative:

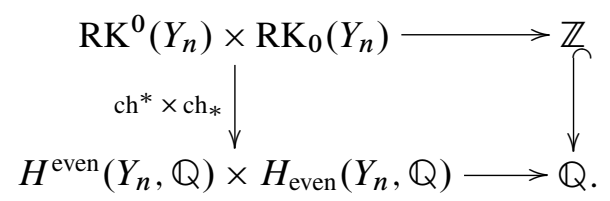

Thus $\left\langle\operatorname{ch}^{*}\left(v_{n}(x)\right), \operatorname{ch}_{*}(z)\right\rangle=\pi_{*}\left(\mu_{n}(z)\right)$. Since $x$ is the class of a unital finite dimensional representation $\pi: C^{*}(G) \rightarrow \mathrm{M}_{n}(\mathbb{C})$, it follows that $v_{n}(x)$ is simply the class of the flat complex vector bundle $[V]=\pi_{*}\left(\ell_{n}\right)$ over $Y_{n}$. On the other hand, if $V$ is a flat vector bundle, then $\operatorname{ch}^{*}(V)=\operatorname{rank}(V)=m=\operatorname{dim}(\pi)$ by [14]. Therefore, for any unital $m$-dimensional representation $\pi, \pi_{*}\left(\mu_{n}(z)\right)=m \cdot\left\langle 1, \operatorname{ch}_{*}(z)\right\rangle$. By applying the same formula for the trivial representation $\iota: C^{*}(G) \rightarrow \mathbb{C}$, we get $\iota_{*}\left(\mu_{n}(z)\right)=\left\langle 1, \operatorname{ch}_{*}(z)\right\rangle$. It follows that $\pi_{*}\left(\mu_{n}(z)\right)=m \cdot \iota_{*}\left(\mu_{n}(z)\right)$.

Recall that we denote by $I(G)$ the kernel of the trivial representation $\iota: C^{*}(G) \rightarrow$ $\mathbb{C}$. Since the extension $0 \rightarrow I(G) \rightarrow C^{*}(G) \rightarrow \mathbb{C} \rightarrow 0$ is split, $K_{0}\left(C^{*}(G)\right) \cong$ $K_{0}(I(G)) \oplus \mathbb{Z}$.

Theorem 3.3. Let $G$ be a countable, discrete, torsion-free group that is uniformly embeddable in a Hilbert space. Let $h: K_{0}\left(C^{*}(G)\right) \rightarrow \mathbb{Z}$ be a group homomorphism.

(i) If $C^{*}(G)$ is $K$-quasidiagonal, then there exist two discrete completely positive asymptotic representations $\left\{\pi_{n}: C^{*}(G) \rightarrow \mathrm{M}_{k(n)}(\mathbb{C})\right\}_{n}$ and $\left\{\gamma_{n}: C^{*}(G) \rightarrow\right.$ $\left.\mathrm{M}_{r(n)}(\mathbb{C})\right\}_{n}$ such that $\pi_{n \sharp}(x)-\gamma_{n \sharp}(x) \equiv h(x)$ for all $x \in \mu\left(\mathrm{RK}_{0}(\mathrm{~B} G)\right)$.

(ii) If $C^{*}(G)$ is $K$-residually finite dimensional, then there is a discrete completely positive asymptotic representation $\left\{\pi_{n}: C^{*}(G) \rightarrow \mathrm{M}_{k(n)}(\mathbb{C})\right\}_{n}$ such that $\pi_{n \sharp}(x) \equiv$ $h(x)$ for all $x \in K_{0}(I(G)) \cap \mu\left(K_{0}(\mathrm{~B} G)\right)$.

Proof. Part (i) follows from Theorem 3.1 and Proposition 2.5 for $A=C^{*}(G)$ and $B=\mathbb{C}$. For part (ii) we observe that if $\gamma_{n}$ is a $*$-representation, then $\gamma_{*}=0$ on $K_{0}(I(G))$ by Proposition 3.2.

Theorem 3.4. Let $G$ be a countable, discrete, torsion-free group. Suppose that $G$ satisfies either one of the conditions (a) or (b) below. 
(a) $G$ has the Haagerup property and $C^{*}(G)$ is $K$-residually finite dimensional.

(b) $G$ is an increasing union of residually finite, amenable groups.

Then for any group homomorphism $h: K_{0}\left(C^{*}(G)\right) \rightarrow \mathbb{Z}$ there is a discrete completely positive asymptotic representation $\left\{\pi_{n}: C^{*}(G) \rightarrow \mathrm{M}_{k(n)}(\mathbb{C})\right\}_{n}$ such that $\pi_{n \sharp}(x) \equiv h(x)$ for all $x \in K_{0}(I(G))$.

Proof. Recall that the assembly map is an isomorphism for groups with the Haagerup property by a result of Higson and Kasparov [12], and that these groups are also embeddable in a Hilbert space. Thus, if $G$ satisfies (a), then the conclusion follows from Theorem 3.3(ii). Suppose now that $G$ satisfies (b). Thus $G=\bigcup_{i} G_{i}$ where $G_{i}$ are residually finite, amenable groups and $G_{i} \subset G_{i+1}$. Then $C^{*}(G)=\overline{\bigcup_{i} C^{*}\left(G_{i}\right)}$ and $K_{0}\left(C^{*}(G)\right) \cong \lim _{0} K_{0}\left(C^{*}\left(G_{i}\right)\right)$. Similarly, $I(G)=\overline{\bigcup_{i} I\left(G_{i}\right)}$ and $K_{0}(I(G))=$ $\lim _{0} K_{0}\left(I\left(G_{i}\right)\right)$. Let $\theta_{i}: K_{0}\left(C^{*}\left(G_{i}\right)\right) \rightarrow K_{0}\left(C^{*}(G)\right)$ be the map induced by the inclusion $C^{*}\left(G_{i}\right) \subset C^{*}(G)$. Let $h$ be given as in the statement of the theorem. By the first part of the theorem, for each $i$, there is a discrete completely positive asymptotic representation $\left(\pi_{n}^{(i)}\right)_{n}$ of $C^{*}\left(G_{i}\right)$ such that $\pi_{n \sharp}^{(i)}(x) \equiv h\left(\theta_{i}(x)\right)$ for all $x \in K_{0}\left(I\left(G_{i}\right)\right)$. By Arveson's extension theorem, each $\pi_{n}^{(i)}$ extends to a unital completely positive map $\bar{\pi}_{n}^{(i)}$ on $C^{*}(G)$. Since $C^{*}(G)$ is separable, $K_{0}(I(G))$ is countable and $K_{0}(I(G))=\lim _{\longrightarrow} K_{0}\left(I\left(G_{i}\right)\right)$, it follows that there is a sequence of natural numbers $r(1)<r(2)<\cdots$ such that $\left(\bar{\pi}_{r(i)}^{(i)}\right)_{i}$ is a discrete completely positive asymptotic representation of $C^{*}(G)$ such that $\bar{\pi}_{r(i), \#}^{(i)}(x) \equiv h(x)$ for all $x \in K_{0}(I(G))$.

\section{Almost flat K-theory classes}

In this section we use the dual assembly to derive the existence of almost flat K-theory classes on the classifying space $\mathrm{B} G$ if the group $\mathrm{C}^{*}$-algebra of $G$ is quasidiagonal. It is convenient to work with an adaptation of the notion of almost flatness to simplicial complexes, see [19].

Definition 4.1. Let $Y$ be a compact Hausdorff space and let $\left(U_{i}\right)_{i \in I}$ be a fixed finite open cover of $Y$. A complex vector bundle $E \in \operatorname{Vect}_{m}(Y)$ is called $\varepsilon$-flat if is represented by a cocycle $v_{i j}: U_{i} \cap U_{j} \rightarrow U(m)$ such that $\left\|v_{i j}(y)-v_{i j}\left(y^{\prime}\right)\right\|<\varepsilon$ for all $y, y^{\prime} \in U_{i} \cap U_{j}$ and all $i, j \in I$. A K-theory class $\alpha \in K^{0}(Y)$ is called almost flat if for any $\varepsilon>0$ there are $\varepsilon$-flat vector bundles $E, F$ such that $\alpha=[E]-[F]$. This property does not depend on the cover $\left(U_{i}\right)_{i \in I}$.

Remark 4.2. The set of all almost flat elements of $K^{0}(Y)$ form a subring denoted by $K_{\mathrm{af}}^{0}(Y)$. If $f: Z \rightarrow Y$ is a continuous map, then $f^{*}\left(K_{\mathrm{af}}^{0}(Y)\right) \subset K_{\mathrm{af}}^{0}(Z)$. 
The following proposition gives a method for producing $\varepsilon$-flat vector bundles. Let $Y$ be a finite simplicial complex with universal cover $\widetilde{Y}$ and fundamental group $G$. Consider the flat line bundle $\ell$ with fiber $C^{*}(G), \widetilde{Y} \times{ }_{G} C^{*}(G) \rightarrow Y$, where $G \subset C^{*}(G)$ acts diagonally, and let $P$ be the corresponding projection in $\mathrm{M}_{m}(\mathbb{C}) \otimes$ $C(Y) \otimes C^{*}(G)$. Consider a discrete asymptotic representation $\left\{\varphi_{n}: C^{*}(G) \rightarrow\right.$ $\mathrm{M}_{k(n)}(\mathbb{C}) \xi_{n}$ and set $F_{n}=\left(\operatorname{id}_{m} \otimes \operatorname{id}_{C(Y)} \otimes \varphi_{n}\right)(P)$. Since $\left\|F_{n}^{2}-F_{n}\right\| \rightarrow 0$ as $n \rightarrow \infty, E_{n}:=\chi\left(F_{n}\right)$ is a projection in $\mathrm{M}_{m k(n)}(C(Y))$ such that $\left\|E_{n}-F_{n}\right\| \rightarrow 0$ as $n \rightarrow \infty$.

Proposition 4.3. For any $\varepsilon>0$ there is $n_{0}>0$ such that for any $n \geq n_{0}$ there is an $\varepsilon$-flat vector bundle on $Y$ which is isomorphic to the vector bundle given by the idempotent $E_{n}$.

Proof. We rely on a construction and results of Phillips and Stone from [20], [21], see also [18]. A simplicial complex is locally ordered by giving a partial ordering $\mathbf{o}$ of its vertices in which the vertices of each simplex are totally ordered. The first barycentric subdivision of any simplicial complex has a natural local ordering [21], \$1.4. Thus we may assume that $Y$ is endowed with a fixed local ordering $\mathbf{o}$. Let $Y$ have vertices $I=\{1,2, \ldots, m\}$. We denote by $Y^{k}$ the set of $k$-simplices of $Y$. Given $r \geq 1$, a $U(r)$-valued lattice gauge field $\mathbf{u}$ on the simplicial complex $Y$ is a function that assigns to each 1-simplex $\langle i, j\rangle$ of $Y$ an element $u_{i j} \in U(r)$ subject to the condition that $u_{j i}=u_{i j}^{-1}$, see [21], Def. 3.2. Consider the cover of $Y$ by dual cells $\left(V_{i}\right)_{i \in I}$ [21], A.1.

Phillips and Stone show that for a fixed locally ordered finite simplicial complex $Y$ as above there is a function $h:[0,+\infty) \rightarrow[0,1]$ with $\lim _{t \rightarrow \infty} h(t)=0$ and which has the following property. Let $\mathbf{u}$ be a $U(r)$-valued lattice gauge field on $Y$ for some $r \geq 1$. Suppose that

$$
\left\|u_{i j} u_{j k}-u_{i k}\right\| \leq \delta
$$

for all 2-simplices $\langle i, j, k\rangle$ (with vertices so o-ordered). Then there is a cocycle $v_{i j}: V_{i} \cap V_{j} \rightarrow U(r),\langle i, j\rangle \in Y^{1}$, such that

$$
\sup _{x \in V_{i} \cap V_{j}}\left\|v_{i j}(x)-u_{i j}\right\|<h(\delta) .
$$

The functions $v_{i j}(x)$ are constructed by an iterative process, based on the skeleton of $Y$. At each stage of the construction one takes affine combinations of functions defined at a previous stage, starting with the constant matrices $u_{i j}$. It follows that for each $i \in I$ there exists a fixed small open tubular neighborhood $U_{i}$ of $V_{i}$ which is affinely homotopic to $V_{i}$, such that the cover $\left(U_{i}\right)_{i \in I}$ has the following property. For any $U(r)$-valued lattice gauge field $\mathbf{u}$ on $Y$ that satisfies (3), there is a cocycle $v_{i j}: U_{i} \cap U_{j} \rightarrow U(r),\langle i, j\rangle \in Y^{1}$, such that

$$
\sup _{x \in U_{i} \cap U_{j}}\left\|v_{i j}(x)-u_{i j}\right\|<2 h(\delta) .
$$


We are going to use the asymptotic representation $\left(\varphi_{n}\right)_{n}$ as follows. Using trivializations of $\ell$ to $U_{i}$ one obtains group elements $s_{i j} \in G$ for $\langle i, j\rangle \in Y^{1}$ giving a constant cocycle on $U_{i} \cap U_{j}$ that represents $\ell$, so that $s_{i j}^{-1}=s_{j i}$ and $s_{i j} \cdot s_{j k}=s_{i k}$ whenever $\langle i, j, k\rangle \in Y^{2}$.

If $\left(\chi_{i}\right)_{i \in I}$ are positive continuous functions with $\chi_{i}$ supported in $U_{i}$ and such that $\sum_{i \in I} \chi_{i}^{2}=1$, then $\ell$ is represented by an idempotent

$$
P=\sum_{i, j \in I} e_{i j} \otimes \chi_{i} \chi_{j} \otimes s_{i j} \in \mathrm{M}_{m}(\mathbb{C}) \otimes C(Y) \otimes C^{*}(G) .
$$

Here $m=|I|$ and $\left(e_{i j}\right)$ is the canonical matrix unit of $\mathrm{M}_{m}(\mathbb{C})$. It follows that for all $n$ sufficiently large, $\left(\operatorname{id}_{m} \otimes \operatorname{id}_{C(Y)} \otimes \varphi_{n}\right)_{\sharp}(P)$ is given by the class of a projection $E_{n}$ with $\left\|E_{n}-F_{n}\right\|<1 / 2$, where $F_{n}=\left(\operatorname{id}_{m} \otimes \operatorname{id}_{C(Y)} \otimes \varphi_{n}\right)(P)$. We have

$$
F_{n}=\sum_{i, j \in I} e_{i j} \otimes \chi_{i} \chi_{j} \otimes \varphi_{n}\left(s_{i j}\right) \in \mathrm{M}_{m}(\mathbb{C}) \otimes C(Y) \otimes \mathrm{M}_{k(n)}(\mathbb{C}) .
$$

For $v \in \mathrm{GL}_{k}(\mathbb{C})$ we denote by $w(v)$ the unitary $v\left(v^{*} v\right)^{-1 / 2}$. Fix $n$ sufficiently large so that $\varphi_{n}\left(s_{i j}\right) \in \mathrm{GL}_{k(n)}(\mathbb{C})$. For each ordered edge $\langle i, j\rangle \in Y^{1}$ we set $u_{i j}=w\left(\varphi_{n}\left(s_{i j}\right)\right)$ and $u_{j i}=u_{i j}^{-1}$. This will define a $U(k(n))$-valued lattice gauge field on the ordered simplicial complex $Y$. Fix $\varepsilon>0$ such that $4 m^{2} \varepsilon<1 / 2$ and choose $\delta>0$ such that $h(\delta)<\varepsilon / 2$. Since $\left(\varphi_{n}\right)_{n}$ is an asymptotic representation, there is $n_{0}>0$ such that if $n \geq n_{0}$, then

$$
\left\|\varphi_{n}\left(s_{i j}\right)-u_{i j}\right\|<\varepsilon / 2
$$

for all $\langle i, j\rangle \in Y^{1}$ and $\left\|u_{i j} u_{j k}-u_{i k}\right\| \leq \delta$ for all 2-simplices $\langle i, j, k\rangle$. By the result of Phillips and Stone quoted above, there exists a cocycle $v_{i j}: U_{i} \cap U_{j} \rightarrow U(k(n))$ such that

$$
\left\|v_{i j}(x)-u_{i j}\right\|<h(\delta)<\varepsilon / 2
$$

for all $x \in U_{i} \cap U_{j}$. It follows that $\left\|v_{i j}(x)-v_{i j}\left(x^{\prime}\right)\right\|<\varepsilon$ for all $x, x^{\prime} \in U_{i} \cap U_{j}$ and all $i, j \in I$ and hence the idempotent

$$
e_{n}(x)=\sum_{i, j \in I} e_{i j} \otimes \chi_{i}(x) \chi_{j}(x) v_{i j}(x), \quad x \in Y,
$$

gives an $\varepsilon$-flat vector bundle on $Y$. From (4) and (5) we have

$$
\left\|v_{i j}(x)-\varphi_{n}\left(s_{i j}\right)\right\|<\varepsilon
$$

for all $x \in U_{i} \cap U_{j}$ and $\langle i, j\rangle \in Y^{1}$. Using (6) we see that $\left\|e_{n}-F_{n}\right\| \leq 2 m^{2} \varepsilon<1 / 2$ and hence $\left\|e_{n}-E_{n}\right\| \leq\left\|e_{n}-F_{n}\right\|+\left\|E_{n}-F_{n}\right\|<1$. It follows that $E_{n}=w e_{n} w^{-1}$ for some invertible element $w$. This shows that the isomorphism class of the vector bundle given the idempotent $E_{n}$ is represented by an $\varepsilon$-flat vector bundle since we have seen that $e_{n}$ has that property. 
Let $Y$ be a finite simplicial complex with universal cover $\tilde{Y}$ and fundamental group $G$ and let $\ell$ be the corresponding flat line bundle with fiber $C^{*}(G)$. Recall that the Kasparov product $K_{0}\left(C(Y) \otimes C^{*}(G)\right) \times \mathrm{KK}\left(C^{*}(G), \mathbb{C}\right) \rightarrow K^{0}(Y)$ induces a map $v: \operatorname{KK}\left(C^{*}(G), \mathbb{C}\right) \rightarrow K^{0}(Y), v(\alpha)=[\ell] \circ(\alpha \otimes 1)$.

Corollary 4.4. $v\left(\mathrm{KK}_{\mathrm{qd}}\left(C^{*}(G), \mathbb{C}\right)\right) \subset K_{\mathrm{af}}^{0}(Y)$.

Proof. This follows from Propositions 2.5 and 4.3.

Theorem 4.5. Let $G$ be a countable, discrete, torsion-free group which is uniformly embeddable in a Hilbert space. Suppose that the classifying space $\mathrm{B} G$ is a finite simplicial complex and that the full group $C^{*}$-algebra $C^{*}(G)$ is $K$-quasidiagonal. Then all the elements of $K^{0}(\mathrm{~B} G)$ are almost flat.

Proof. We have seen in the proof of Theorem 3.1 that under the present assumptions on $G$ the dual assembly map $v: \operatorname{KK}\left(C^{*}(G), \mathbb{C}\right) \rightarrow K^{0}(\mathrm{~B} G)$ is surjective. Since $C^{*}(G)$ is K-quasidiagonal by hypothesis (this holds for instance if $C^{*}(G)$ is quasidiagonal as observed in Remark 2.3), we have $\mathrm{KK}\left(C^{*}(G), \mathbb{C}\right)=\mathrm{KK}_{\mathrm{qd}}\left(C^{*}(G), \mathbb{C}\right)$. The result follows now from Corollary 4.4 .

From Theorem 4.5 one can derive potential obstructions to quasidiagonality of group $\mathrm{C}^{*}$-algebras.

Remark 4.6. Let $G$ be a countable, discrete, torsion-free group which is uniformly embeddable in a Hilbert space and such that the classifying space $\mathrm{B} G$ is a finite simplicial complex. If not all elements of $K^{0}(\mathrm{~B} G)$ are almost flat, then $C^{*}(G)$ is not quasidiagonal.

\section{References}

[1] F. P. Boca, A note on full free product $C^{*}$-algebras, lifting and quasidiagonality. In Operator theory, operator algebras and related topics (Timişoara, 1996), Theta Found., Bucharest 1997, 51-63. Zbl 0942.46036 MR 1728412

[2] X. Chen, M. Dadarlat, E. Guentner, and G. Yu, Uniform embeddability and exactness of free products. J. Funct. Anal. 205 (2003), 168-179. Zbl 1041.46043 MR 2020212

[3] P.-A. Cherix, M. Cowling, P. Jolissaint, P. Julg, and A. Valette, Groups with the Haagerup property. Progr. Math. 197, Birkhäuser, Basel 2001. Zbl 1030.43002 MR 1852148

[4] A. Connes, M. Gromov, and H. Moscovici, Conjecture de Novikov et fibrés presque plats. C. R. Acad. Sci. Paris Sér. I Math. 310 (1990), 273-277. Zbl 0693.53007 MR 1042862

[5] A. Connes and N. Higson, Déformations, morphismes asymptotiques et $K$-théorie bivariante. C. R. Acad. Sci. Paris Sér. I Math. 311 (1990), 101-106. Zbl 0717.46062 MR 1065438 
[6] M. Dadarlat, On the asymptotic homotopy type of inductive limit $C^{*}$-algebras. Math. Ann. 297 (1993), 671-676. Zbl 0791.46047 MR 1245412

[7] M. Dadarlat and S. Eilers, On the classification of nuclear $C^{*}$-algebras. Proc. London Math. Soc. (3) 85 (2002), 168-210. Zbl 1031.46070 MR 1901373

[8] M. Dădărlat and T. A. Loring, $K$-homology, asymptotic representations, and unsuspended E-theory. J. Funct. Anal. 126 (1994), 367-383. Zbl 0863.46045 MR 1305073

[9] R. Exel and T. A. Loring, Invariants of almost commuting unitaries. J. Funct. Anal. 95 (1991), 364-376. Zbl 0748.46031 MR 1092131

[10] R. Exel and T. A. Loring, Finite-dimensional representations of free product $C^{*}$-algebras. Internat. J. Math. 3 (1992), 469-476. Zbl 0809.46055 MR 1168356

[11] B. Hanke and T. Schick, Enlargeability and index theory. J. Differential Geom. 74 (2006), 293-320. Zbl 1122.58011 MR 2259056

[12] N. Higson and G. Kasparov, $E$-theory and $K K$-theory for groups which act properly and isometrically on Hilbert space. Invent. Math. 144 (2001), 23-74. Zbl 0988.19003 MR 1821144

[13] T. G. Houghton-Larsen and K. Thomsen, Universal (co)homology theories. K-Theory 16 (1999), 1-27. Zbl 0918.19005 MR 1673935

[14] F. Kamber and P. Tondeur, The characteristic homomorphism of flat bundles. Topology 6 (1967), 153-159. Zbl 0147.42401 MR 0208620

[15] G. G. Kasparov, Equivariant $K K$-theory and the Novikov conjecture. Invent. Math. 91 (1988), 147-201. Zbl 0647.46053 MR 0918241

[16] G. G. Kasparov and G. Skandalis, Groups acting on buildings, operator $K$-theory, and Novikov's conjecture. K-Theory 4 (1991), 303-337. Zbl 0738.46035 MR 1115824

[17] A. Lubotzky and Y. Shalom, Finite representations in the unitary dual and Ramanujan groups. In Discrete geometric analysis, Contemp. Math. 347, Amer. Math. Soc., Providence, RI, 2004, 173-189. Zbl 1080.22006 MR 2077037

[18] V. M. Manuilov and A. S. Mishchenko, Almost, asymptotic and Fredholm representations of discrete groups. Acta Appl. Math. 68 (2001), 159-210. Zbl 1012.46061 MR 1865957

[19] A. S. Mishchenko and N. Teleman, Almost flat bundles and almost flat structures. Topol. Methods Nonlinear Anal. 26 (2005), 75-87. Zbl 1093.19005 MR 2179351

[20] A. Phillips and D. Stone, Lattice gauge fields, principal bundles and the calculation of topological charge. Comm. Math. Phys. 103 (1986), 599-636. Zbl 0597.53065 MR 832541

[21] A. V. Phillips and D. A. Stone, The computation of characteristic classes of lattice gauge fields. Comm. Math. Phys. 131 (1990), 255-282. Zbl 0718.55012 MR 1065672

[22] A. I. Shtern, Finite-dimensional quasirepresentations of connected Lie groups and Mishchenko's conjecture. Fundam. Prikl. Mat. 13 (2007), No. 7, 85-225; English transl. J. Math. Sci. 159 (2009), 653-751. Zbl 1187.22014 MR 2475577

[23] G. Skandalis, Une notion de nucléarité en $K$-théorie (d'après J. Cuntz). $K$-theory 1 (1988), 549-573. Zbl 0653.46065 MR 0953916

[24] G. Skandalis, J. L. Tu, and G. Yu, The coarse Baum-Connes conjecture and groupoids. Topology 41 (2002), 807-834. Zbl 1033.19003 MR 1905840 
[25] J.-L. Tu, La conjecture de Baum-Connes pour les feuilletages moyennables. $K$-Theory 17 (1999), 215-264. Zbl 0939.19001 MR 1703305

[26] J.-L. Tu, The gamma element for groups which admit a uniform embedding into Hilbert space. In Recent advances in operator theory, operator algebras, and their applications, Oper. Theory Adv. Appl. 153, Birkhäuser, Basel 2005, 271-286. Zbl 1074.46049 MR 2105483

[27] D. Voiculescu, Asymptotically commuting finite rank unitary operators without commuting approximants. Acta Sci. Math. (Szeged) 45 (1983), 429-431. Zbl 0538.47003 MR 708811

[28] D. Voiculescu, Around quasidiagonal operators. Integral Equations Operator Theory 17 (1993), 137-149. Zbl 1048.47501 MR 1220578

[29] G. Yu, The coarse Baum-Connes conjecture for spaces which admit a uniform embedding into Hilbert space. Invent. Math. 139 (2000), 201-240. Zbl 0956.19004 MR 1728880

Received February 16, 2012]

M. Dadarlat, Department of Mathematics, Purdue University, 150 N. University Street, West Lafayette, IN 47907-2067, U.S.A.

E-mail: mdd@math.purdue.edu 